\title{
Use of Modified Polyacrylamide for Floccuation of Coal Enrichment Wastes
}

\author{
Vasyl Duleba, Zoriana Gnativ \\ Department of Chemical Endineering, Lviv Polytechnic National University, UKRAINE, Lviv, 12 S.Bandera street, \\ E-mail: zoriana.y.hnativ@lpnu.ua
}

Abstract - In various industrial processes it is necessary to separate liquid and solid phases (condensation, filtration, centrifugation, flotation). Water purification from suspended particles concentration, phase separation in suspension having big problems caused by small particle size and high aggregative and sedimentation stability of the system. Intensification of these processes in many cases can be achieved at the expense of consolidation of particles in aggregates under the action of coagulants and flocculants, and especially their mixtures.

Keywords - separation suspension, flocculant, coagulant, polyacrylamide.

\section{Introduction}

Properties of technical polyacrylamides are widely described in the literature. They are widely used in water treatment, industrial waste water purification, flocculation and flocculation flotation (for flotation with pre-aggregation with the help of flocculants) of a large number of mineral suspensions - in the extraction of minerals from various ores, in coal extraction, in the food industry, etc.

The suspension of solid phase precipitation at application of modified flockulants on the basis of polyacrylamide-50 and PAAM-52 obtained in our laboratory were investigated. PAM50 is a polyacrylamide, modified using a mixture of epoxy fatty acids. PAM-52 is a polyacrylamide, partly crosslinked Formaldegìdom, leading to an increase in the molecular mass of polyacrylamide, and, consequently, increases the rate of solid deposition of suspension tails of flotation while reducing the cost of flockulants. However, in the case of use for separation of suspension tails, the flotation of coal enrichment is more effective in the PAAM-50. Its application allows to get the highest rate of deposition of solid phase and to get the most clean drain on the thickness.

\section{Conclusion}

Study of the influence of a PATC expenditure on the rate of deposition of clay particles at different concentrations of solid phase suspension showed that with the concentration of solid phase up to $15 \mathrm{~g} / \mathrm{L}$ deposition rate grows to $7.5 \mathrm{~mm} / \mathrm{s}$. decrease of solid phase concentration Leads to a decrease of deposition rate of clay particles, which can be explained by the formation of smaller-sized floules because the distance between the particles increases sharply.

This effect shows that at the concentration of a solid share of more than $20 \mathrm{~g} / \mathrm{L}$ is the most productive conditions for floccuation, that is, at lower costs polyacrylamide per mass unit of solid phase there is formation of the flocle of optimum size.

\section{References}

[1] L. A. Kul's`ky`j and P. P. Strokach, Texnology`ya ochy`stky` pry`rodnux vod, Ky`ev: Vy`shha shkola, 1981.

[2] R. P. Singh, P. Chang, G. V. Reddy, ets. "Charakteristiks of so polimer, polimer-fibre combinations and grafied polymers as drag reducing agents and their industrial application “, Drag Reduct.3 ${ }^{\text {rd }}$ Int Conf. Bristol, 1984, pp. D4/1-D4/5. 